\title{
Evidence of Decreasing Internet Entropy: The Lack of Redundancy in DNS Resolution by Major Websites and Services
}

\author{
SAMANTHA BATES \\ Harvard Law School, United States of America \\ JOHN BOWERS \\ Yale Law School, United States of America \\ SHANE GREENSTEIN \\ Harvard Business School, United States of America \\ JORDI WEINSTOCK \\ Harvard Law School, United States of America \\ YUNHAN XU \\ Harvard College, United States of America \\ JONATHAN ZITTRAIN \\ Harvard Law School \& Harvard School of Engineering and Applied \\ Sciences, United States of America
}

The Internet, and the Web built on top of it, were intended to support an "entropic" physical and logical network map (Zittrain, 2013). That is, they have been designed to allow servers to be spread anywhere in the world in an ad hoc and evolving fashion, rather than a centralized one. Arbitrary distance among, and number of, servers causes no particular architectural problems, and indeed ensures that problems experienced by one data source remain unlinked to others. A Web page can be assembled from any number of upstream sources, through the use of various URLs, each pointing to a different location. To a user, the page looks unified. Over time, however,

Greenstein: sgreenstein@hbs.edu

Date submitted: 2020-12-25

Copyright (C) 2021 (Samantha Bates, John Bowers, Shane Greenstein, Jordi Weinstock, Yunhan Xu, Jonathan Zittrain). Licensed under the Creative Commons Attribution-NonCommercial-NoDerivatives 4.0 International Public License. Available at: http://journalqd.org 
there are signs that the hosting and finding of Internet services has become more centralized. We explore and document one possible dimension of this centralization. We analyze the extent to which the Internet's global domain name resolution (DNS) system has preserved its distributed resilience given the rise of cloud-based hosting and infrastructure. We offer evidence of the dramatic concentration of the DNS hosting market in the hands of a small number of cloud service providers over a period spanning from 2011-2018. In addition, we examine changes in domains' tendency to "diversify" their pool of nameservers - how frequently domains employ DNS management services from multiple providers rather than just one provider. Throughout the paper, we use the catastrophic October 2016 attack on Dyn, a major DNS hosting provider, to illustrate the cybersecurity consequences of our analysis.

Keywords: domain name system, resilience, cybersecurity, entropy

On October 21, 2016, it appeared to many worldwide Internet users that the network had broken. Users in North America or Europe trying to access major websites such as Netflix, CNBC, and Twitter found only blank screens or error messages. It soon became clear that the cause was a distributed denial of service (DDoS) attack against Dyn, a major domain name system (DNS) service provider. ${ }^{1}$ Dyn reported that its servers were overwhelmed by a flood of requests from a botnet of "Internet of Things" (IoT) devices infected by Mirai malware (Hilton, 2016).

As a DNS provider, Dyn enables Internet traffic by translating the site's domain name (URL) into the IP address where the server behind that domain is to be found. Website operators often contract with Dyn and other companies like it to manage that "lookup" process rather than handling it themselves. During the attack, Dyn servers were

\footnotetext{
${ }^{1}$ Note that Dyn was acquired by Oracle in 2016, and currently operates as a business unit within Oracle. However, we refer to it as "Dyn" throughout this paper for clarity's sake.
} 
unable to process users' lookup requests (both legitimate and illegitimate), and as a result, users lost access to web domains reliant on Dyn such as Netflix, CNBC, and Twitter (Newman, 2016). While it is impossible to calculate the economic cost of such an attack, Dyn itself has claimed that " $[\mathrm{t}]$ he cost of a DDoS attack can quickly reach up to million dollars an hour in lost revenue and productivity for the average size company" (Dyn, 2017).

The Dyn attack illustrates how fragile the Internet can be when malicious actors know how to exploit its vulnerabilities. The Internet comprises many servers, traditionally found in a wide variety of physical and logical locations. An ethos of decentralization is woven into its central protocols - they anticipate and embrace the messiness of an organically-growing network unconstrained by any sort of central plan. This tendency towards decentralization, which we refer to as the internet's "entropy," is the secret to its resilience and ability to deal with failure. When the internet's functionality is distributed across a vast sea of physically and logically distinct hosts, single points of failure are minimized.

However, the internet's decentralized ideal has more and more fallen out of alignment with its reality. The past decade has seen a surge in cloud hosting: the servers that store and distribute the content hosted by a website are more and more likely to reside in a massive server farm owned by one of a handful of dominant cloud providers rather than in an on-site server room. And while it offers massive economies of scale as well as advantages in reliability, performance, and expertise, this more centralized hosting model has undermined the internet's "entropic" resilience. With centralization comes single points of failure - if a major provider goes offline, so too might the broad swath of websites and services that rely upon it (Albert et al., 2000).

DNS services, which are often part-and-parcel of website hosting arrangements, have followed this trajectory of centralization - and now confront the vulnerabilities associated with it. While the Dyn attack represents one dramatic example of how a loss of 
entropy across internet infrastructure exposes Internet-wide vulnerabilities exploitable by bad actors, we doubt it will be the last such incident.

This paper examines the increasing concentration of the DNS hosting industry. We argue that a long-term shift towards centralization of DNS hosting - to the benefit of a small handful of cloud services providers like Dyn, Amazon Web Services, and Cloudflare - has, along with website administrators" failure to designate failover "secondary" DNS providers, created dangerous single points of failure in the DNS. We use the Dyn attack as a running case study to illustrate that these single points of failure present a significant cybersecurity risk, and suggest some paths forward.

Part I offers an overview of the DNS for those less familiar with its technical specifications, using the Dyn attack to illustrate how the DNS can fail. Part II describes the article's empirical methodology. Part III presents results and analysis relating to the increased concentration of the DNS hosting market, observing that market share has become concentrated in the hands of a small number of dominant firms. Part IV offers an empirical examination of website administrators' widespread failure to designate secondary DNS hosts, a measure which could function as a fallback in the event that the primary host fails. Parts III and IV both illustrate cybersecurity risks related to their observations using the Dyn attack. Part V concludes.

\section{What is the Domain Name System?}

DNS servers perform a variety of functions that make them an integral part of the Internet's infrastructure. This paper will focus primarily on DNS's role as a "website directory" or authoritative resolver which translates a website's human-friendly domain name (like www.example.com) into a machine-friendly IP address (like 192.0.2.1) pointing to the location of the website's host server on the Internet. The information needed 
to complete these translations is stored in definitive form by a domain's "authoritative nameservers" in files called "resource records" (RRs). ${ }^{2}$

DNS is structured as a hierarchically distributed database. At the very top of the hierarchy are 13 "root" servers. ${ }^{3}$ Administered by a range of organizations including governments and branches of the US military, these root servers are responsible for storing RRs corresponding to Top-Level Domain (TLD) nameservers. A vast range of TLDs (including ".com," ".net," ".org," and country-level identifiers such as ".uk" or ".fr") can be found at the rightmost end of the domains we access every day. TLD nameservers are managed by a wide range of public and private organizations under authority delegated by the Internet Corporation for Assigned Names and Numbers. Each TLD nameserver - or network thereof - is responsible for keeping RRs corresponding to the authoritative nameservers ${ }^{4}$ of domains that fall within that TLD. ${ }^{5}$ For example, the ".com" nameservers keep RRs for the authoritative nameservers for the domain namespace encompassing domain names such as "www.google.com" and "www.amazon.com."

Authoritative nameservers for a given domain namespace can be administered by that domain's owners, or management can be outsourced to one or more external providers such as Dyn, AWS, or Cloudflare. They form the last stage in the DNS hierarchy, storing RRs that provide translations between domain names and IP addresses.

\footnotetext{
2 There are many different types of resource records, but for the sake of simplicity we do not distinguish between them.

${ }^{3}$ More precisely, 13 networks of root servers with internal redundancy.

${ }^{4}$ In practice, there can be additional non-authoritative nameservers between the TLD nameserver and the domain's authoritative nameserver. In such cases, the hierarchy is simply extended accordingly. For example, when requesting the IP address of https://www.oii.ox.ac.uk/, the resolver may be directed to the nameservers for ' $u k$ ', 'ac' and 'ox' before it reaches the domain's authoritative nameserver.

5 These sets of RRs corresponding to a particular domain are often referred to as "NS records."
} 


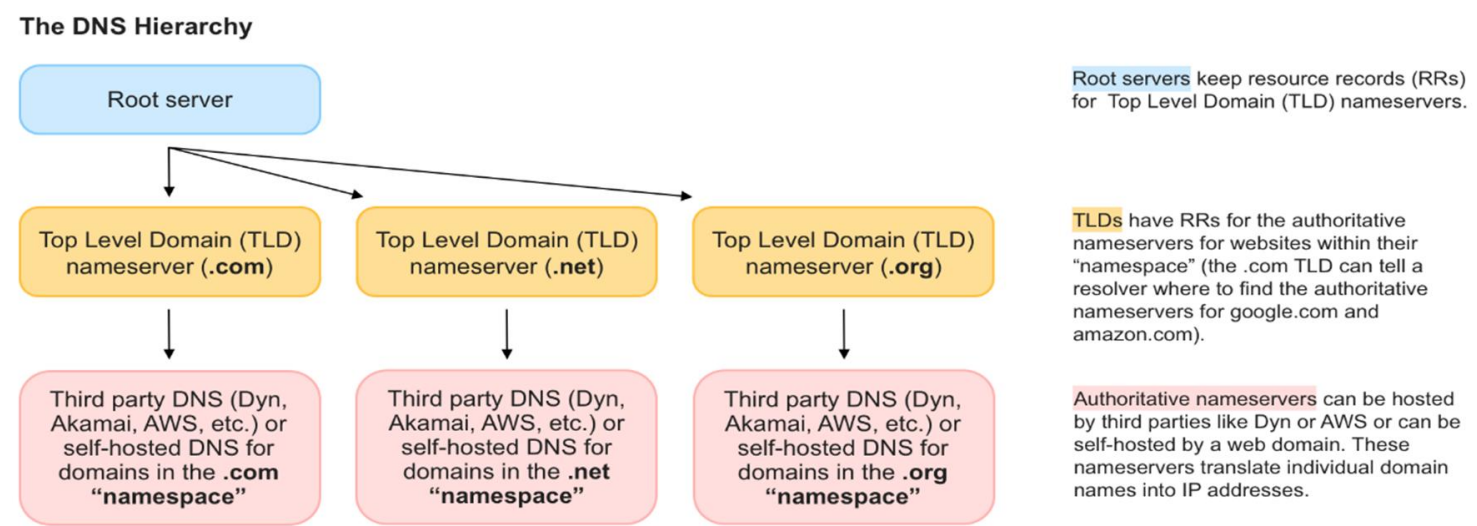

Figure 1. The DNS hierarchy.

\section{Tracing a DNS Request}

Understanding how the DDoS attack on Dyn interfered with this process means tracing through the steps of a DNS lookup. When an application (such as a web browser) wants to access a page or resource located at a known domain name, it can leverage the DNS system to find a corresponding IP address. In principle, the application submits a request to a DNS "resolver" asking for the IP address corresponding to a given domain name, specified in URL format (ex. "www.google.com"). The resolver traces through this URL's period-separated components from right to left in order to zero in on the desired authoritative nameserver.

The resolver first queries a root nameserver, which replies with RRs corresponding to the TLD nameserver specified by the domain name (".com"). The resolver then queries that specified TLD nameserver with the second component of the domain name ("google"). The TLD nameserver retrieves the RRs corresponding to that domain's authoritative nameservers ("nsl.google.com") and returns them to the resolver. Finally, the resolver queries one of the authoritative nameservers and receives a usable IP address for the domain. The IP address is passed back to the original application, which can use it to connect to the desired host. This entire process generally takes just milliseconds to finish. 


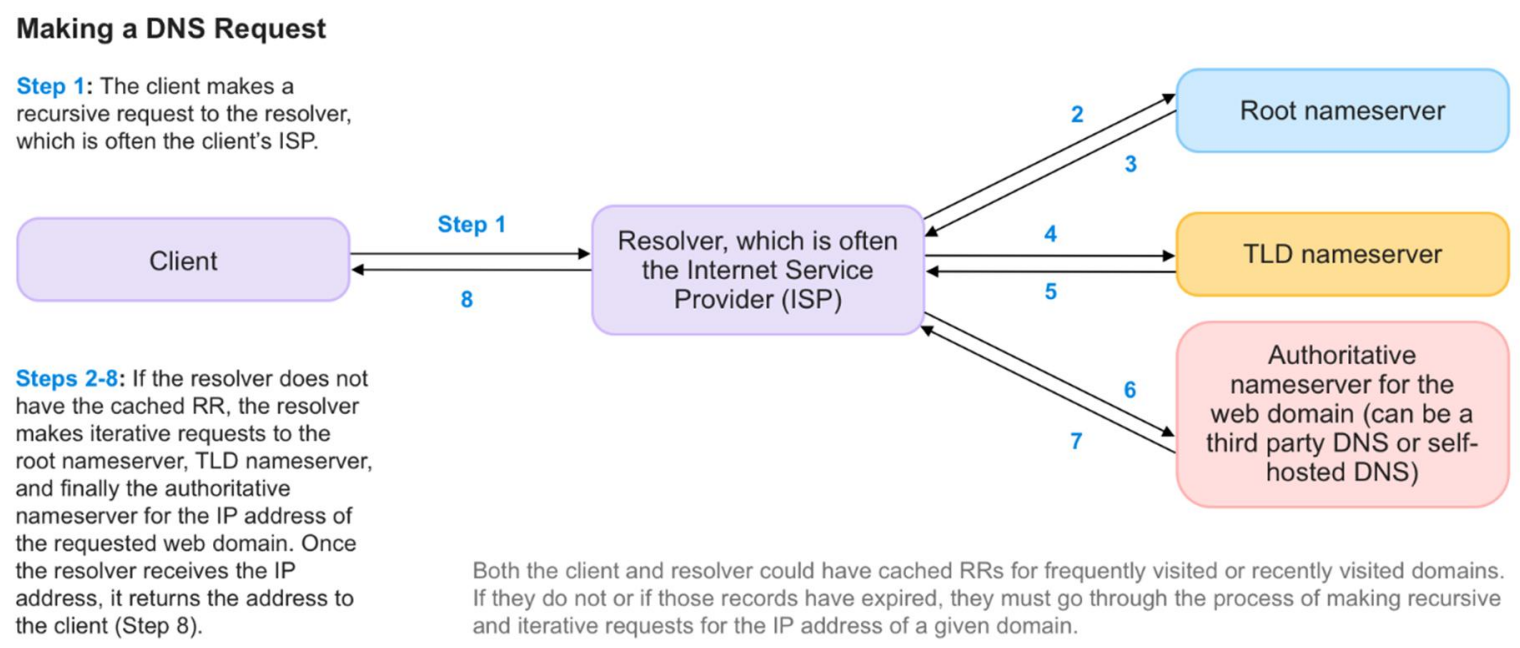

Figure 2. Making a DNS request.

If every DNS request passed through all of these intermediary stages, the DNS infrastructure would face a constant and potentially overwhelming deluge of traffic. Luckily, RRs are cached at numerous points in the lookup process. Applications and Internet-connected devices maintain small caches of RRs for recently visited sites, as do Internet Service Providers (ISPs) and other intermediate nameservers. Of course, the content of these records can become inaccurate over time as IPs change. As such, RRs eventually expire. Most RRs must be replaced after twenty-four hours, though many larger sites implement much shorter expiration periods. ${ }^{6}$ The duration for which nameservers are allowed to cache a RR without updating it is that RR's 'time to live' (TTL). An RR with a short TTL will have to be updated more often, but it is also more likely to remain accurate (Liu \& Albitz, 2006, Chapter 2).

If the authoritative nameserver corresponding to a particular domain name goes down (as many of those administered by Dyn did in the October DDoS attack), DNS resolvers become unable to update RRs that have expired or changed. In the event of a DNS failure IP addresses may still resolve to the correct host, but it is very unlikely that

\footnotetext{
${ }^{6}$ For example, the "dig" command, a tool for querying DNS servers reveals that the RRs for www.spotify.com had a TTL of 150 seconds as of October 12th, 2020.
} 
any user will know the exact current IP addresses of the websites they want to access. As such, a DNS malfunction effectively prevents users from accessing the content they have requested even if that content is hosted on an otherwise healthy server.

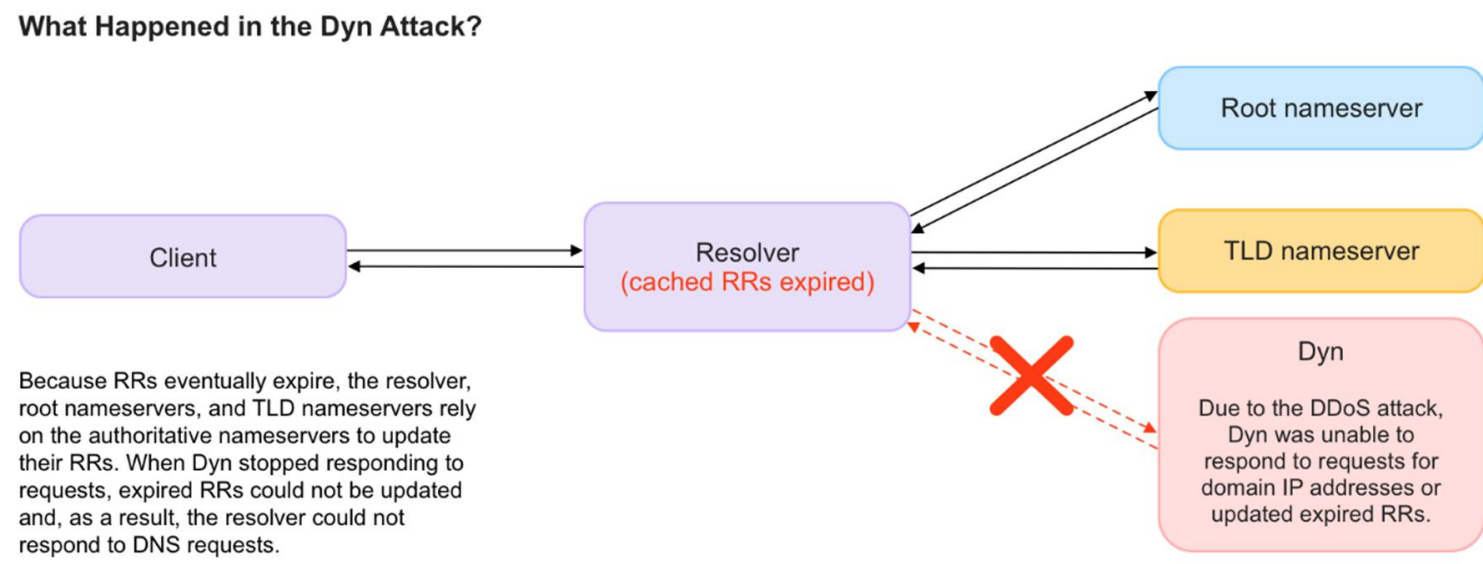

Figure 3. What happened in the Dyn attack?

\section{DNS: Designed for Resilience?}

As is evident from the hierarchical architecture outlined above, the global Domain Name System (DNS) infrastructure is distributed by design (Mockapetris \& Dunlap, 1988). Rather than being handled by a single master server mapping domain names to IP addresses, DNS lookups rely on interactions among millions of different servers worldwide. This distributed model theoretically brings a degree of segmentation and redundancy to the DNS system, minimizing "single points of failure" at which technical breakdowns result in access problems for significant swaths of the Internet (Kurose \& Ross, 2013, Chapter 2.5).

However, the rise of cloud-based hosting and domain management services threatens to overturn this distributed model of resilience. Companies such as Amazon Web Services (Amazon, 2021), Akamai (Akamai, 2021), and Dyn offer scalable and often easily configurable external DNS hosting options alongside other cloud services, making it easier 
than ever to offload DNS management. External DNS hosting can offer significant advantages in terms of load balancing, reliability, and geographic reach. As of 2017, Dyn's DNS service boasted points of presence on five continents, a team of dedicated security experts, a sophisticated performance analytics engine, and a multitude of load balancing and traffic steering features (Dyn, 2017). ${ }^{7}$ Moreover, large and centralized DNS providers have the means and incentives to invest in cybersecurity readiness to an extent that would be infeasible for most of their individual clients.

Alongside these advantages, however, the consolidation of DNS services for a vast range of web domains into the hands of a relatively small number of providers could potentially pose a threat to the stability of the Internet. With consolidation comes single points of failure that create opportunities for simultaneous downtime. As in the case of the Dyn attack, the reachability of many domains can easily hinge on the resilience and stability of a single monolithic provider. Moreover, if the DNS market moves towards an oligopolistic or monopolistic structure, the lack of competition among DNS providers may decrease the diversity of providers available to consumers. Such a market shift could escalate consolidation further while stifling consumer-friendly business practices and making DNS servers even more attractive targets to malicious actors.

The fact that externally hosted DNS providers offer high quality service while concentrating the DNS hosting industry presents an important tradeoff between two different patterns of downtime. On one hand, having a relatively small number of large externally hosted DNS providers with significant market share generates single points of failure which expose large segments of the Internet to simultaneous downtime. On the other, externally hosted DNS providers are - as mentioned above - generally very successful in maintaining almost perfect uptime while provisioning a range of sophisticated additional services that can substantially improve DNS performance. A tradeoff thus

\footnotetext{
${ }^{7}$ In addition to translating domain names into IP addresses, DNS servers can also be used to direct and balance Internet traffic so that no individual server is burdened by too many requests. These types of functions are called load balancing and traffic steering. For the purposes of this paper we investigate the impact of concentration only on DNS's role as a "website directory."
} 
emerges between occasional periods of widespread downtime and potentially more frequent but highly distributed patterns of DNS downtime for individual sites or smaller externally hosted DNS providers.

\section{The Stakes of DNS Resilience}

The Dyn attack provides a vivid illustration of how DNS infrastructure vulnerabilities - and DNS space concentration - can wreak havoc on the stability of the Internet. Prompting a widespread Internet outage that left many high-profile websites inaccessible for a period of hours (Perlroth, 2016), the attack's devastating success highlights many of the ways in which a concentrated DNS space with relatively little use of secondary DNS providers can leave even well-resourced and well-managed websites vulnerable to service disruptions.

Why, though, does DNS downtime matter? Is it really a big deal if a swath of the Internet becomes inaccessible for a few hours every now and then? In short, yes. Even brief periods of downtime can have a dramatic impact on the economic well-being of affected companies. A 2014 survey of 270 North American companies with a significant web presence found that about $50 \%$ of DDoS attacks cost their targets more than $\$ 20,000$ an hour, with 49\% of attacks lasting between six and twenty-four hours (Matthews, 2014). Some larger companies can suffer more than $\$ 100,000$ an hour in IT, security, and sales related damages. About $87 \%$ of companies that had been targeted by a DDoS attack reported "at least one non-financial consequence such as loss of customer trust, loss of intellectual property, and virus/malware infection” (Matthews, 2014).

DNS downtime has a range of other less explicitly economic impacts on a company's web presence. Search engines use web crawlers, automated scripts that can be used to copy visited websites or search for specific information, to index pages and 
determine search result presentation precedence. ${ }^{8}$ If a site is unreachable during a crawl due to DNS downtime, the crawler will mark it as having been "uncrawlable" at the time of attempted access - a designation with potentially devastating consequences for a site's visibility ("How DDoS Attacks Can Impact Your SEO," 2013). Furthermore, since DNS is involved in handling services such as a domain's email functionality, DNS downtime can cripple communications within and between companies.

The DNS infrastructure will face more pressure from malicious actors in the coming years, not less. An annual report published by the cybersecurity company Nexusguard found that in Q1 2017 the number of DNS attacks they observed "registered a $380 \%$ year-on-year growth, suggesting that DDoS attacks occurred more frequently than the same period a year ago" (Nexusguard, 2017). The report partially attributes the proliferation of massive DDoS-capable botnets to the rise of the so-called "Internet of Things," which continues to bring an enormous number of often poorly secured webconnected devices online. The "Mirai" botnet which brought Dyn to its knees is only one example of such a weapon. As more and more devices are added to the global network, the number and power of botnets will almost certainly increase (Nozomi Networks, 2020). DNS resilience is not simply about protecting against today's DDoS attacks - it is also about anticipating and preparing for tomorrow's.

Of course, it doesn't always take hundreds of thousands of unsecured webcams and Internet-connected toasters or other IoT devices to bring down a cloud services behemoth. In the course of routine maintenance in early 2017, a tech at Amazon accidentally caused a number of Amazon web servers to go offline (Seppala, 2017). The server failure impacted services like Slack, Quora, and Medium that rely on Amazon cloud storage (Fingas, 2017). The AWS status page failed to properly reflect the outage due to hard dependencies on the service that it was designed to monitor (@awscloud, 2017).With

\footnotetext{
${ }^{8}$ Web crawlers are automated scripts or programs that search the web for specific information (usually for indexing purposes) in a systematic way.
} 
consolidation comes the risk that simple mistakes on the part of engineers and administrators will cascade into widespread outages.

\section{Methodology}

To analyze trends in the concentration of the DNS hosting industry over time, as well as the rate at which websites designate secondary DNS providers, we sampled the top 1000 U.S. domains in the ".com," ".net," or ".org" Top-Level Domains (TLDs) according to Alexa Top Sites (Alexa, n.d.) listings on a monthly basis between November 2011 and July 2018. We recognize that our results could change if we included domains from other regions, such as ".cn" and ".ru," in our dataset. ${ }^{9}$ However, we chose to focus on traditional domains, ".com," ".net," and ".org" because they are among the longest standing TLDs and comparatively represent a broad "general purpose" sample of high-traffic Internet sites. Due to our sampling method, the set of domains examined each month varied depending

\footnotetext{
${ }^{9}$ Sites using "Country Code TLDs" like ".cn" and ".ru" are typically hosted outside of the United States, and tend to cater to a non-U.S. audience. For example, Alibaba Cloud and Tencent Cloud are significant players in the Chinese hosting ecosystem, but barely show up in our analysis. As such, the results here tend to focus in on the dynamics of the U.S. DNS hosting industry in particular. That said, the TLDs examined here account for a large proportion of the domains registered globally (173.9 million of the 370.7 million identified in a 2020 Verisign analysis) and most of the largest and most visited sites on the Internet.
} 
on the composition of the Alexa rankings. ${ }^{10}$ This sampling methodology is also approximate because our dataset is missing about $4.5 \%$ of rankings per month. ${ }^{11}$

The motivation for this approach is drawn from the use of market indexes in economics - indexes such as the S\&P500 are composed to reflect the performance and behavior of a broader market (Blitzer, 2013). It is important to note that DNS providers' market share in this sample does not reflect that of the broader Internet - our focus is on high-traffic domains which are far less likely to use low-end-market oriented providers such as GoDaddy DNS (Datanyze).

\footnotetext{
${ }^{10}$ Alexa Top Sites data has some deficiencies, but was the best available dataset for our study. One drawback of the dataset is that Alexa's ranking algorithm is not transparent. See (Alexa, 2020) However, it should be noted that other ranking companies also prefer to keep their ranking algorithms secret and it is often not possible to obtain verified data from any of these companies. Moreover, the top ranked sites identified by Alexa, Quantcast, Comscore, and other ranking companies are comparable, confirming that our dataset of top ranked sites is generally reflective of the consensus among analysts. While there may be differences among rankings depending on the company, these differences tend to occur most frequently at the extreme end of samples (sites outside of the top 1000).

We do not have access to Alexa Top Sites data from after July 2018. We explored using a list from the Tranco team, which began publishing top sites rankings in December of 2018 (Tranco, 2020). Integrating the Tranco data yielded results broadly consistent with those presented here, despite some significant differences in the specific compositions of the Alexa and Tranco lists. However, we did note that domains using Cloudflare as their DNS provider tended to rank far less well on the Tranco list compared to the Alexa Top Sites list, and that the list reflected significant turnover in domain rankings from March 2020 to the present, potentially driven by changes in user behavior arising from the COVID-19 pandemic. A paper published by the team behind the Tranco list offers some important insights into the volatility and manipulability of top sites lists in general, and the implications of those vulnerabilities for academic research (Le Pochat et al., 2019). We deeply appreciate the work of the Tranco team, which is taking on one of the most serious methodological issues confronting quantitative internet research today.

${ }^{11}$ These missing data are seemingly distributed in completely random fashion and are therefore highly unlikely to bias the results of our analysis. However, this random variation do introduce a small amount of additional "jitter" into the trendlines presented here. We experimented with imputing missing values based on the previous and following months, which had a very slight smoothing effect, but ultimately elected to use the data as collected.
} 


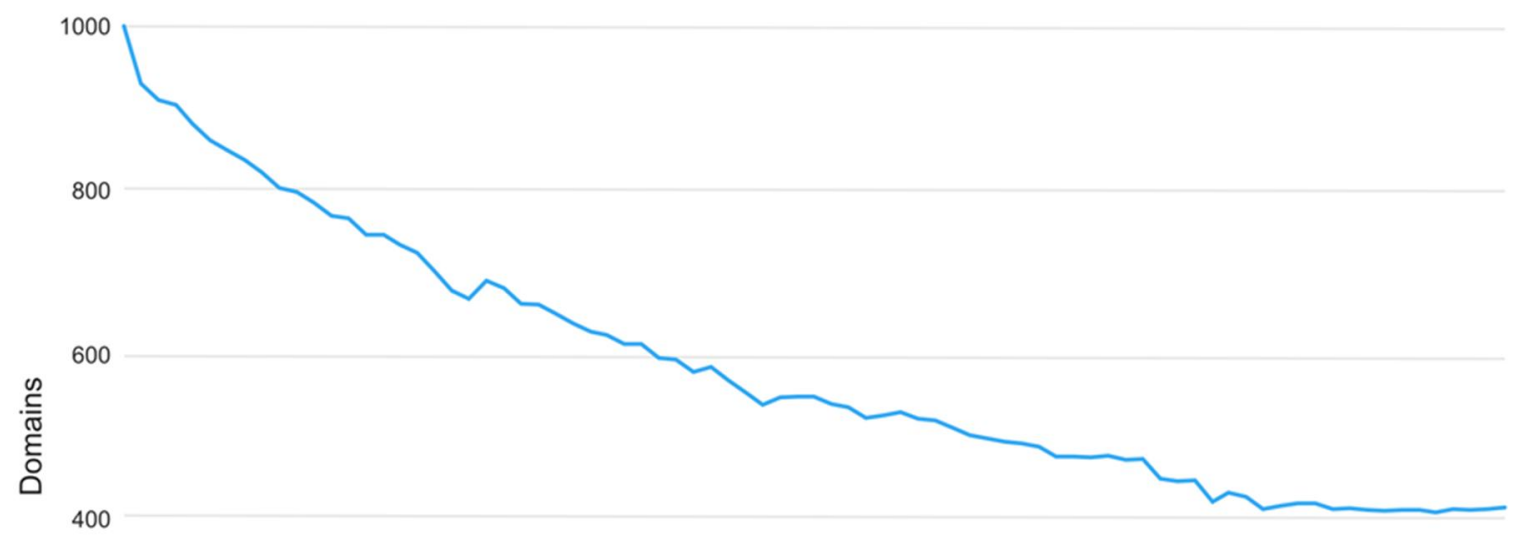

200

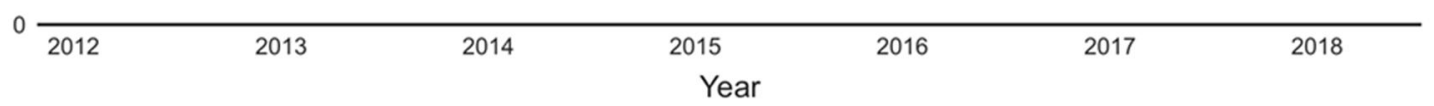

Figure 4. Original top-1000 domains in the top 1000 sites (Alexa ranking) over time.

It is also challenging to determine the importance and representativeness of a domain because it is not as easy to quantitatively model as that of a publicly traded entity. While the contributions of companies included in a conventional stock market index might be weighted using relatively straightforward and publicly available metrics such as market capitalization, weighting domains is not nearly so simple. Even seemingly basic metrics such as page views per month are very difficult to estimate accurately without insider analytics (Fishkin, 2015). However, despite these challenges, we believe that our data are representative of most high-traffic Internet sites within the TLDs examined. Given that "eyeball share" on the Internet is highly concentrated within a relatively small set of sites, sampling 1000 of the most popular sites on the Internet every month captures a sample which represents a large proportion of all Internet behavior in the U.S.

We collected historical nameserver information for each of the domains identified in our dataset using an API offered by CompleteDNS. These data record changes in each 
domain's nameserver registration on a monthly basis, allowing us to identify migrations to and away from external DNS providers or self-hosted DNS solutions. We cross-checked our historical nameserver information against a number of comparable providers and found no evident discrepancies or errors. We were not concerned with how much of a domain's DNS traffic was routed through a given nameserver. Rather, we looked at the number of distinct authoritative nameservers registered by a domain and which providers managed those nameservers. By combining our monthly sample of domains with these historical nameserver data, we were able to generate a month-by-month timeline of nameserver configurations for our sample.

\section{Concentration of the DNS Hosting Market}

The extent to which an industry's providers is concentrated has major implications for that industry's vulnerability to catastrophic wide-ranging failure. If a large proportion of share in the market served by the industry is divided among a small number of providers, outages affecting any one of those providers may have dramatic repercussions for the industry's customers as a whole. In other words, high-share providers have the potential to become single points of failure for large segments of the Internet. In examining the concentration of the DNS hosting market, we aimed to shed light on the extent to which it falls prey to these vulnerabilities.

This section presents our analysis demonstrating that the DNS hosting market has become significantly more concentrated since November of 2011. It concludes by examining the security implications of that concentration, using the Dyn attack as a case study.

\section{DNS Hosting as a Market}

Our sampling methodology defines a set of high-traffic domains under the ".com," ".net," and ".org" TLDs. Within this set we can observe, using the methodology outlined 
above, continuities and changes in the DNS nameservers delegated by each domain in the sample, domains' DNS usage, and how domains adjust their DNS architectures over time. Each domain in our sample uses one or more DNS providers. Some domains host and manage their own DNS nameservers, in which case they are their own DNS provider. Others rely entirely on external providers such as Dyn, Amazon Web Services (AWS), and Akamai. At any given time, a domain may have multiple nameservers (generally between two and eight) managed by a combination of providers or by a single provider.

We define the DNS hosting market as the set of providers - including external DNS providers like Dyn, Akamai, and AWS, and others, as well as "self-hosting" domains which run their own servers - handling DNS for our sample of domains. A provider's share of the market is defined as the number of domains in the sample for which that provider handles at least one nameserver. The total size of the market is defined as the number of domains or "fractional domains" 12 administered by each provider summed across all providers present in the sample.

\section{Concentration of the DNS Market as a Whole}

To quantitatively model the concentration of the DNS hosting market over time we adopted a metric from the antitrust economics literature: the Herfindahl-Hirschman Index (HHI). ${ }^{13}$ A standard measure of market concentration used by the Department of Justice, Federal Trade Commission, and Census bureau, the HHI is defined as the sum of squared

\footnotetext{
${ }^{12}$ In cases where a domain's DNS services is diversified among multiple providers, share is divided evenly among all providers used. For example, a domain managed by two providers would contribute 0.5 share to each. In economics, share in these cases is generally divided according to proportions of sales $-\mathrm{a}$ comparable division metric unfortunately does not exist in this case.

${ }^{13}$ The Herfindahl-Hirschman Index has faced criticism for its inability to capture subtler forms of market consolidation, and its failure to account for market-specific nuances of competition policy (like the unique competitive dynamics of the utilities industry). In some sense, its greatest strength - its simplicity and interpretability - is also its critical weakness (Matsumoto et al., 2011). That said, we are using the HHI here primarily as a heuristic to uncover the sign and magnitude of the trend towards consolidation in the DNS market, so the metric's deficiencies, while worth keeping in mind, are not prohibitive. We supplement it with CR1, CR4, and CR8 metrics to reinforce our analysis.
} 
market shares across all firms in a market (Rhoades, 1993). It ranges from 0 (for a perfectly distributed market) to 10,000 (a perfectly concentrated market). More formally:

$$
H H I=\sum_{i=1}^{N} s_{i}{ }^{2}
$$

Where $\mathrm{N}$ is the number of firms (DNS providers in our case) and $s_{i}$ is the market share for the $i$-th firm.

The HHI is generally used to measure market share in terms of revenues collected by suppliers; however, that is not the case here. Because we are limited by the information that is publicly available (we do not have access to the pricing of services or the revenues collected by the suppliers in this market), the HHI in this paper measures market share based on supplier choice without accounting for revenue. Applying the HHI to our dataset revealed a considerable degree of consolidation within the DNS hosting market between November 2011 and July 2018.

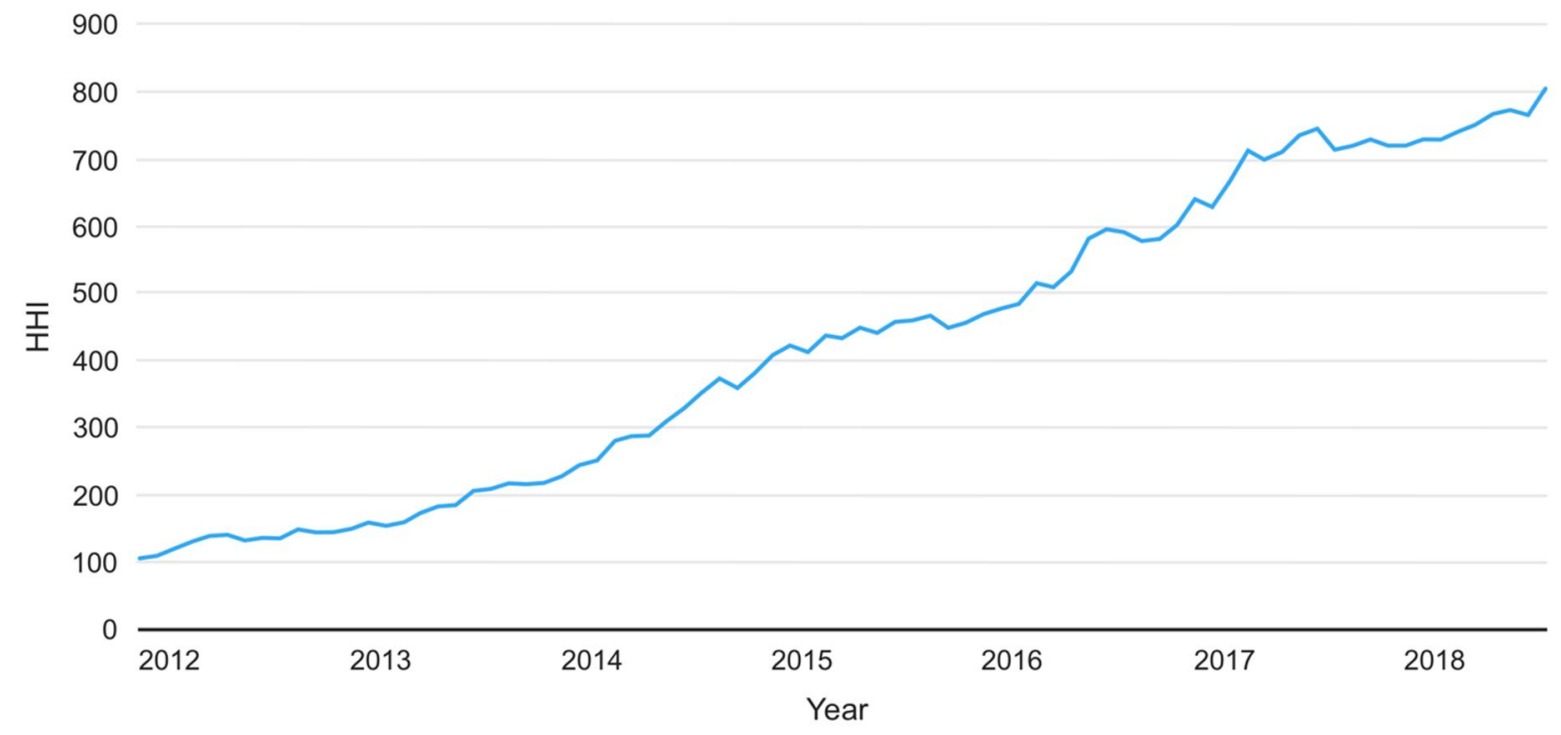

Figure 5. HHI of DNS providers in our dataset by year. 
As is apparent from figure 5 (pictured above), the HHI increased by a factor of about 7.5 over the observed timespan. ${ }^{14}$ This indicates that a number of DNS service providers managed to significantly increase their proportional share of the DNS hosting market in that timeframe, beginning to consolidate control of DNS services. The linearity of the trend is striking - gains in concentration have been relatively consistent in the long run despite yearly fluctuations.

To better understand the nature of this share capture, we looked at the percentage of share belonging to the top provider, top 4 providers, and top 8 providers in the DNS space over time (note that top providers occasionally changed on a month-by-month basis). As is reflected in figure 6 below, a small number of providers have come to largely dominate the DNS hosting market. The percentage of share held by the top 8 providers more than doubled between November 2011 and July 2018, increasing from about 24\% to about $61.6 \%$. The percentage of share held by the top 4 providers grew by an even greater proportion, increasing from about $17 \%$ to about $51.8 \%$. The top provider in the sample controlled $4.9 \%$ of share in November 2011 and $18.9 \%$ of share in July 2018.

An analysis of share over time for several of these top providers offers further insight into changes in the DNS hosting market. The figures below shows change in market share over time for all providers that were in the top 3 (ranked by market share) at any point in the sample timeline. Note that a number of rapidly expanding providers including Dyn, Akamai, AWS, and Cloudflare have captured a large proportion of the DNS hosting

\footnotetext{
${ }^{14}$ In recognition of the fact that a disproportionate volume of internet activity is dominated by a few major websites - Google, Facebook, Youtube, etc. - we recalculated the HHI using a weighting scheme so as to represent the outsized influence of these top websites. All domains' share in the sample were weighted by a factor of $w(R)=e^{-2.6545-\ln (R)}$ (where $R$ is the domain's Alexa rank), a function derived from the regression of a domains' share of global traffic on its Alexa rank presented by Shiller et al. (DOI:10.3386/w23058). This weighting scheme had pronounced effects on the HHI: it grew from 794 in November 2011 to 1107 in July 2018, experiencing frequent fluctuations due to small changes in the ordering of the top few sites. Dropping the top 5 domains from the dataset after weighting caused the HHI to grow from 262 in November 2011 to 600 in July 2018, and dropping the top 25 domains caused the HHI to grow from 138 in November 2011 to 642 in July 2018, much more in line with the unweighted trend illustrated above.
} 
market while other players such as Neustar and DNSPod have retained significant share over time.

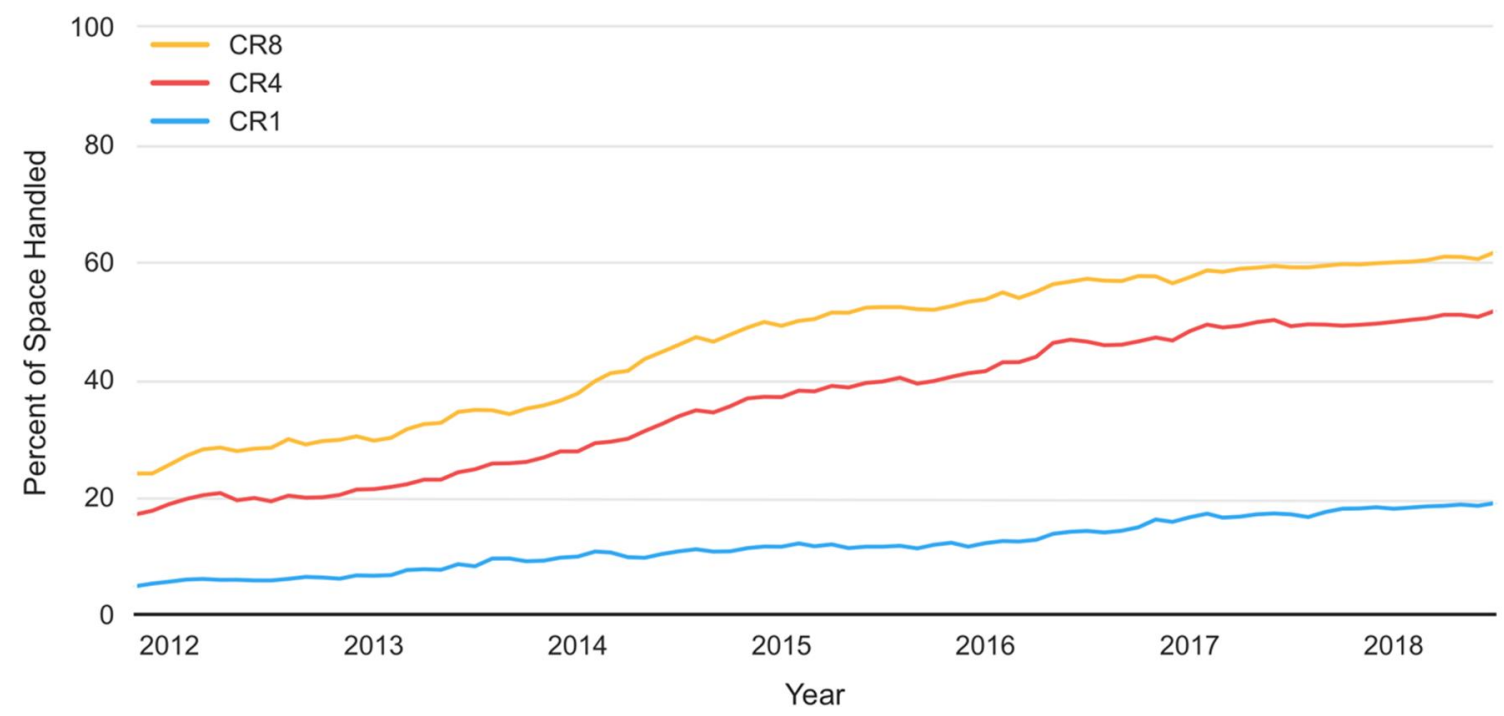

Figure 6. Percentage of the DNS market controlled by the top DNS providers.

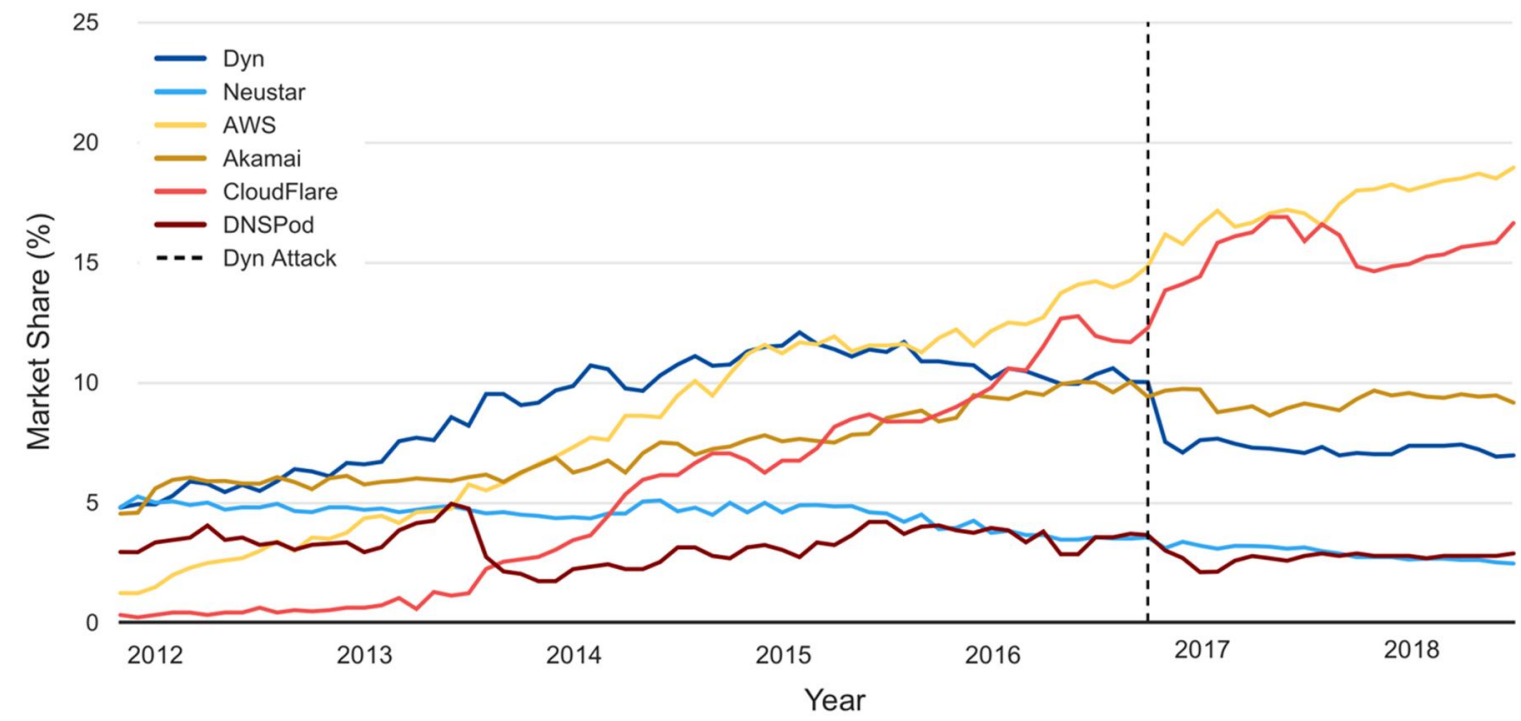

Figure 7. Market share of the top DNS providers. 
It is also worth noting that domains often use these providers for more than just DNS, as many also offer services including Content Delivery Network (CDN) assistance and site hosting.

Domains that entered our sample after November 2011 tended to use external DNS hosting at a much higher rate than those present in the original sample. The DNS hosting market for entrants showed a July 2018 HHI value of over 1100, as opposed to about 600 for the DNS hosting market for domains present in the original sample. This signals that newer domains which have broken into the high-traffic sphere may be relying more and more on a specific set of external DNS hosts.

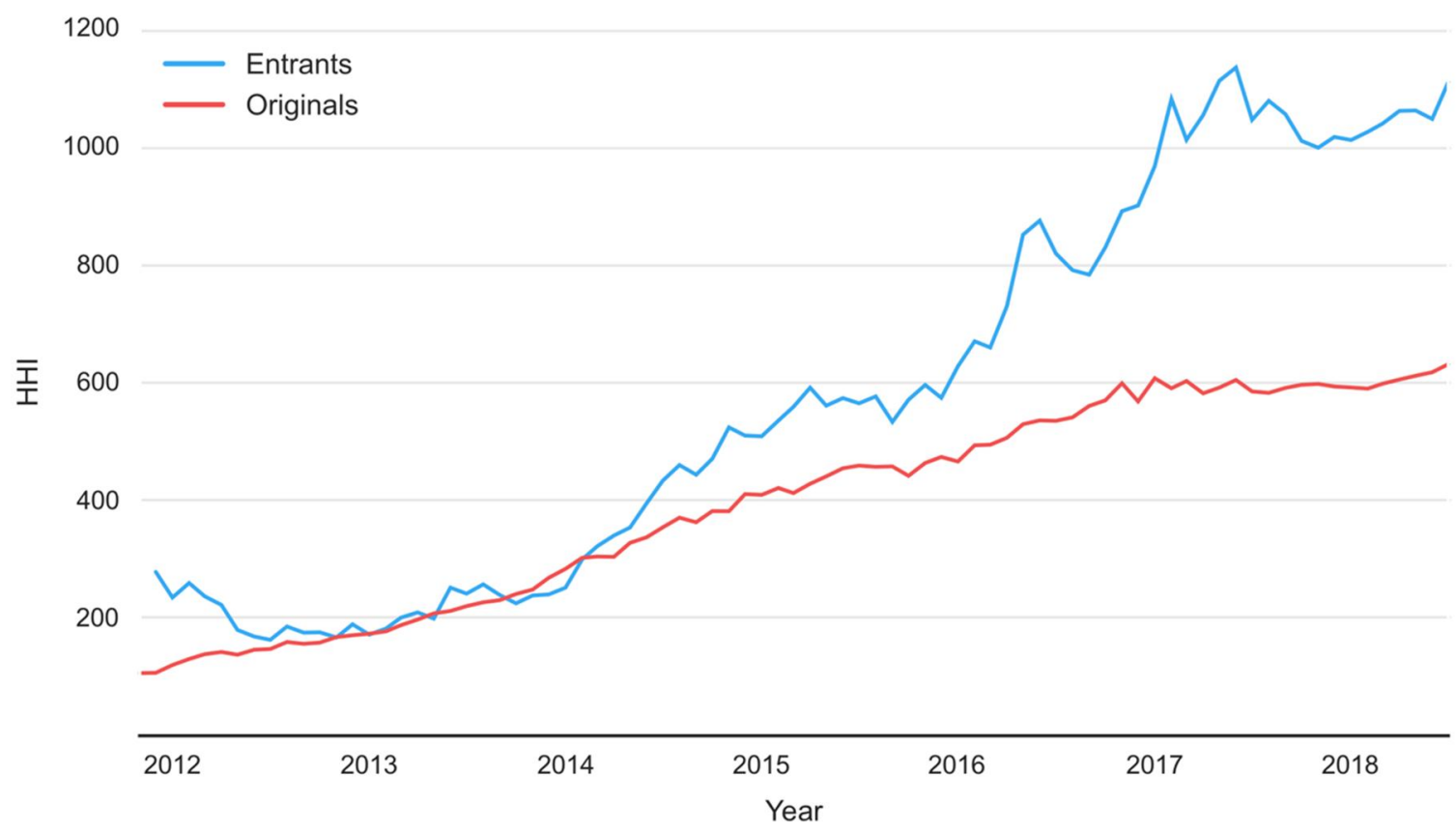

Figure 8. The HHI of original and entrant domains over time.

\section{Concentration of Self-Hosted vs. Externally Hosted DNS}

While the HHI gives us a means of conceptualizing the DNS hosting market in terms of existing market analysis frameworks, DNS is distinct from most conventional 
markets in that many domains choose to host and administer their own DNS nameservers rather than use external hosting providers such as Dyn or Cloudflare. Companies that host their own DNS nameservers may maintain a server on their property or host their nameservers with a service like Rackspace under their own names and remain responsible for managing it. By differentiating external hosting providers from self-hosting providers - domains managing their own DNS nameservers - we can both identify trends in the frequency of self-hosting and analyze the external hosting market independently.

Our analysis of self-hosting and external hosting reveals that concentration of the DNS hosting market is driven by two primary forces - the concentration of the external hosting space into the hands of a small number of providers and a widespread migration away from self-hosted DNS towards externally hosted DNS. The rise of external hosting promotes concentration by enabling the consolidation of market share that would otherwise be distributed among a large number of self-hosts into the hands of a single entity.

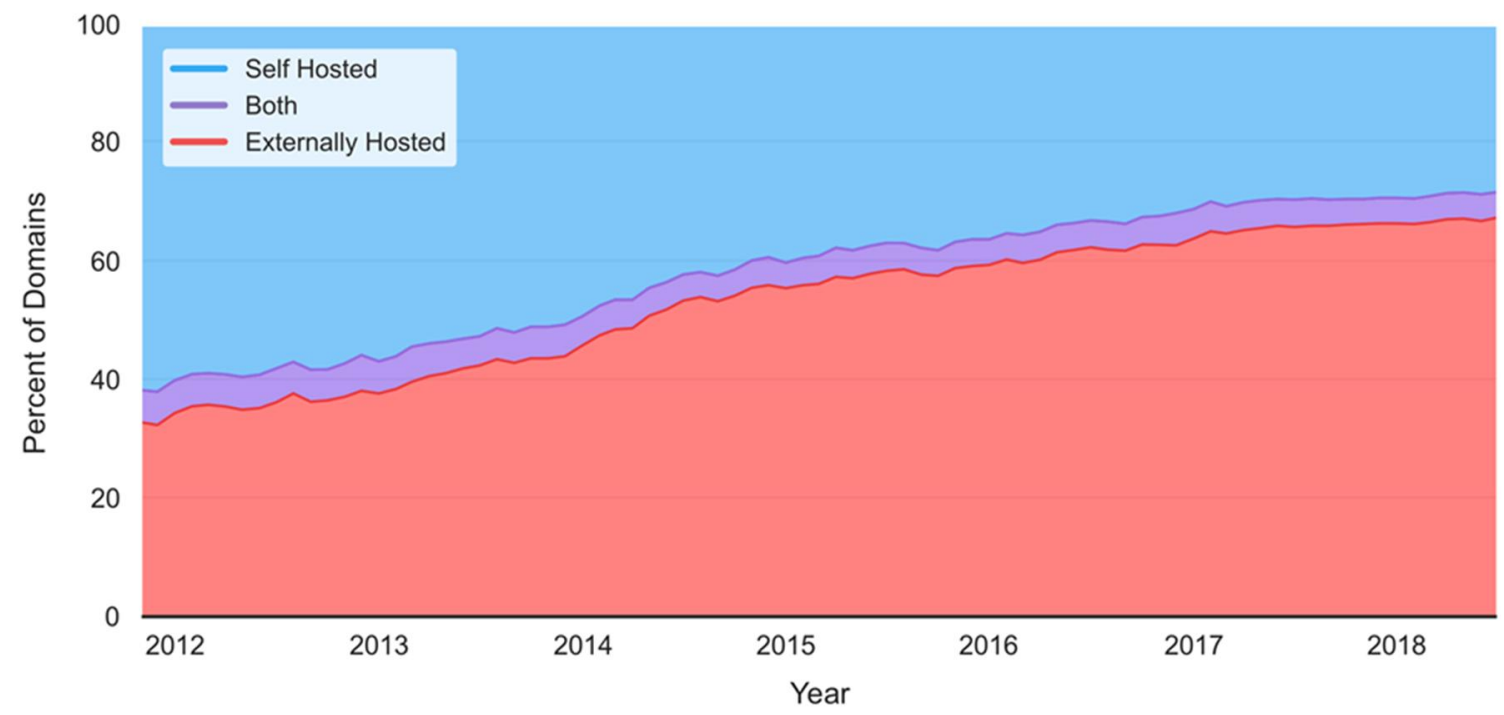

Figure 9. Percentage of self-hosted vs. externally hosted domains over time. 
As is evident from figure 9, external DNS hosting rapidly overtook self-hosted DNS in the period between November 2011 and July 2018. ${ }^{15}$ The percentage of domains managed entirely by external DNS hosting providers grew from $32.9 \%$ to $67.5 \%$ over that period. By looking at the concentration of the two market segments independently, we can better pinpoint the sources of increased space-wide concentration. The HHI of the external hosting market segment more than doubled between November 2011 and July 2018 as a small number of providers took an increasing proportion of share. The external hosting market segment is massively more concentrated than the self-hosting market segment, with HHI averaging about 1181 over the timespan as opposed to about 47 for the self-hosting space. As of July 2018, it stood at about 1622, an increase from 730 in November 2011.

\section{0}

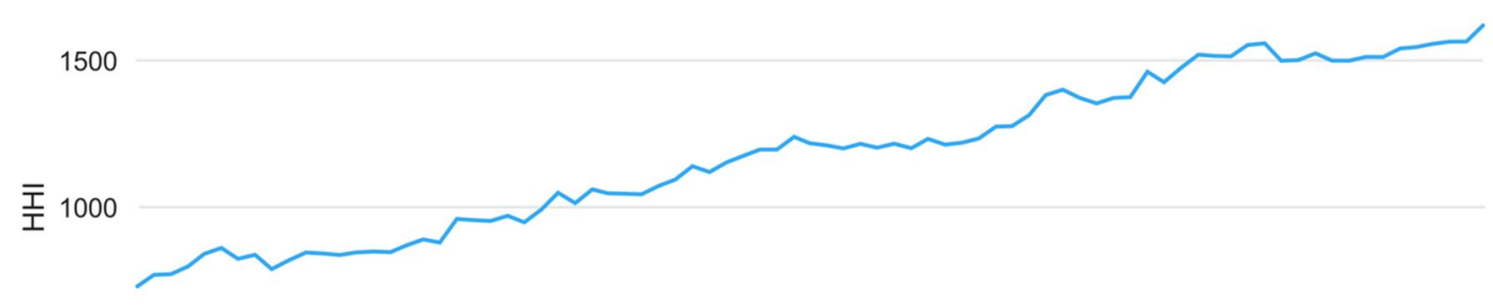

500

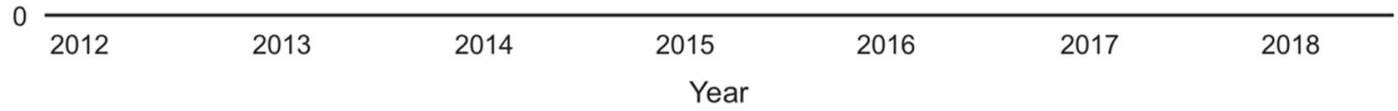

Figure 10. The HHI of the external DNS hosting market.

In sum, the increased concentration of the DNS hosting market can be said to be driven by two simultaneously occurring phenomena: the consolidation of the external

\footnotetext{
${ }^{15}$ Rerunning this analysis under the weighting scheme described in footnote 35 gave significantly different results: after weighting, the percentage of domains using externally hosted DNS services grew from $19.8 \%$ in November 2011 to $30.8 \%$ in July 2018. The lesser magnitude of this change is explained by leading and heavily weighted - domains' proclivity towards self-hosting: Google's self-hosted domains account for about $25 \%$ of total weighted share in the sample throughout the timeline, but less than $1 \%$ of the unweighted sample.
} 
hosting market segment and the migration of domains out of the self-hosting market segment into the external hosting market segment. In other words, more and more domains are choosing to delegate DNS management to an increasingly dominant handful of cloud hosting services. This shift is concurrent with - and driven by - a broader movement towards cloud-based site hosting and management platforms, which is particularly observable among newer websites.

\section{The Dyn Attack: Dangers of DNS Concentration}

The Dyn attack offers a dramatic example of the security consequences of the concentration of the DNS hosting market. At the time of the attack, Dyn held about 10\% share in the overall DNS hosting market. Having expanded from holding $4.8 \%$ share in November 2011, Dyn was among the biggest DNS providers in the sample - a major winner of the process of consolidation described above. Its clients included Twitter, Amazon, Zillow, SoundCloud, and eBay among many others.

When Dyn's systems went offline during the Mirai botnet's DDoS attack, they took most of that $10 \%$ with them - as well as numerous other services with dependencies on Dyn-managed websites and infrastructure.

The dramatic consequences of the Dyn attack illustrate the extent to which DNS space consolidation has exposed large swaths of the Internet to shared single points of failure. Had fewer domains relied solely on Dyn's DNS services, the DDoS attack would have been far less catastrophic. If large external DNS hosting providers continue to absorb market share from smaller competitors and from the self-hosted segment of the DNS space, this non-distributed exposure will only become more pronounced. While large, specialized DNS hosts may fail less often than self-hosting arrangements, they make much juicier targets for bad actors - and when they fail, the consequences are system-wide and catastrophic. 


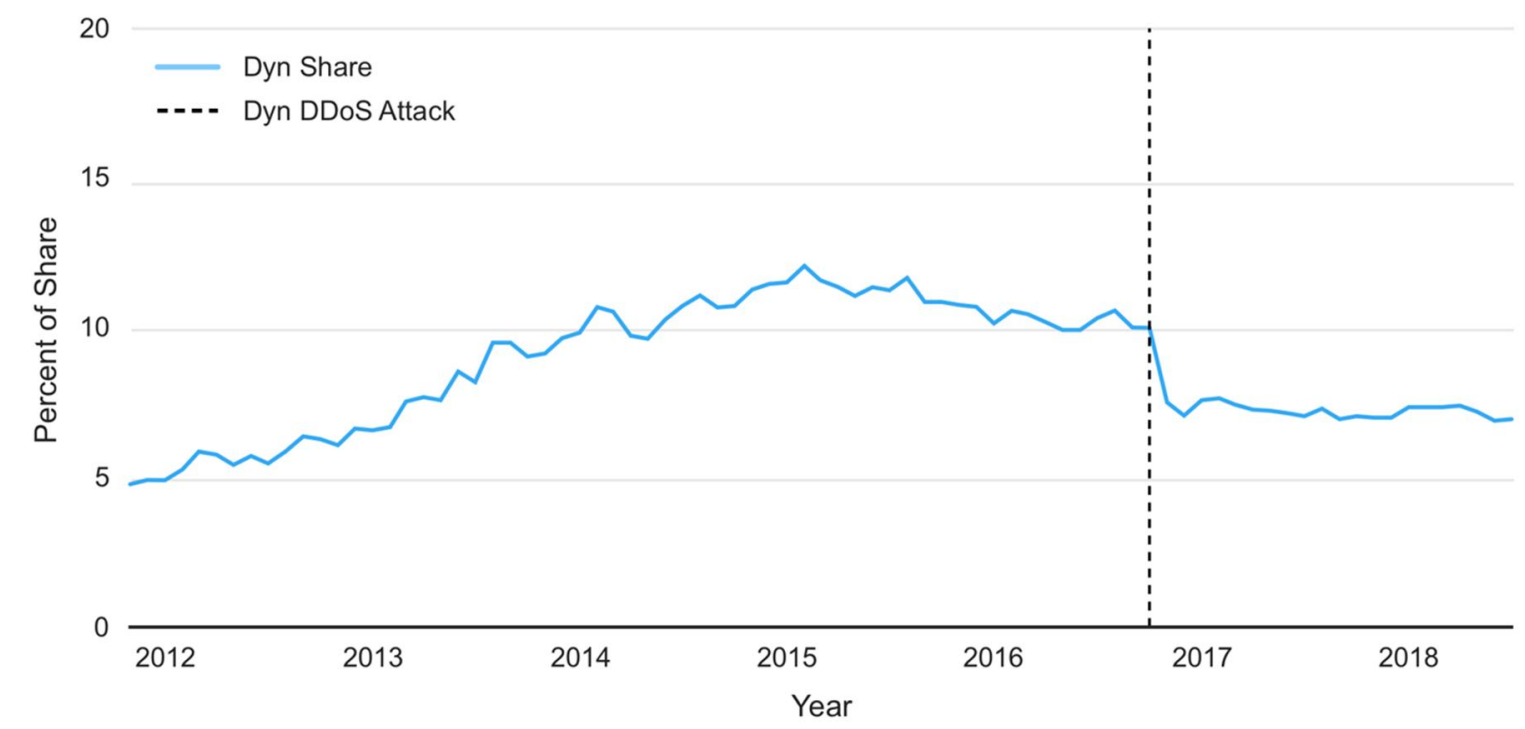

Figure 11. Dyn's share of the DNS hosting market over time.

\section{Secondary DNS: An Overlooked Safeguard}

While the concentration of the DNS space as outlined above has the potential to expose dangerous single points of failure in the DNS system, a powerful safeguard against such vulnerabilities is built into the specification of DNS itself. By allowing domains to register multiple nameservers, DNS gives potentially vulnerable domains the opportunity to diversify among multiple providers. For example, if you registered the domain www.example.com, you could then choose to register more than one authoritative nameserver for that domain, with each authoritative nameserver managed by a different provider. One of your nameservers could be managed by Dyn, example.Dyn.1, and a second could be managed by Akamai, example.Akamai.1. In the event of another DDoS attack on Dyn, users would still be able to access www.example.com as long as your DNS nameserver managed by Akamai remained up and running - the query would "fail over" to the secondary provider. We refer to the adoption of one or more alternative DNS providers as "DNS diversification." 
The importance of DNS redundancy and diversification is not a new realization. RFC 2182, last updated in 1997, outlines best practices for "selection and operation of secondary DNS servers." It explains that "a major reason for having multiple servers for each zone is to allow information from the zone to be available widely and reliably to clients throughout the Internet, that is, throughout the world, even when one server is unavailable or unreachable" (Elz, R. et al., 1997). Similarly, our findings suggest that by registering DNS servers managed by multiple DNS providers, a domain will remain accessible in the event that one provider fails due to an attack or technical malfunction because it is unlikely that both providers will go down at the same time. By choosing to work with multiple providers, any domain can therefore secure significant redundancy and robustness even in an increasingly concentrated DNS space.

Our results suggest, however, that a large majority of domains in our sample are not taking advantage of this capability. This section presents analysis demonstrating strikingly low rates of secondary DNS usage across the sample, despite a strong uptick following the Dyn attack, and relates that phenomenon to cybersecurity principles illustrated by the Dyn attack.

\section{Use of DNS Diversification}

From November 2011 to October 2016, the proportion of domains in our sample using nameservers from just one provider fluctuated in the range between $91 \%$ and $93 \%$. At no point in our timeframe did $1 \%$ or more of domains use 3 or more providers.

As the figures above illustrates, the Dyn attacks of October 2016 have seemingly spurred and sustained interest in DNS diversification. ${ }^{16}$ From October 1st, 2016 to

\footnotetext{
${ }^{16}$ When domains diversify, they may do so in a number of ways. For instance, a domain may choose to delegate DNS services between two or more externally hosted DNS providers. A firm working with Dyn might, for example, choose to add additional nameservers from Akamai or AWS to diversify their DNS. Alternately, a firm that manages its own DNS might choose to diversify with secondary services from a major externally hosted DNS provider or vice versa. As of July 2018 , about $65.6 \%$ of diversified domains
} 
November 1st, 2016, the percentage of domains using a single DNS provider fell from $92.2 \%$ to $89.4 \%$. The percentage continued to decline between November 2016 and July 2018 , reaching $87.1 \%$ in July 2018.

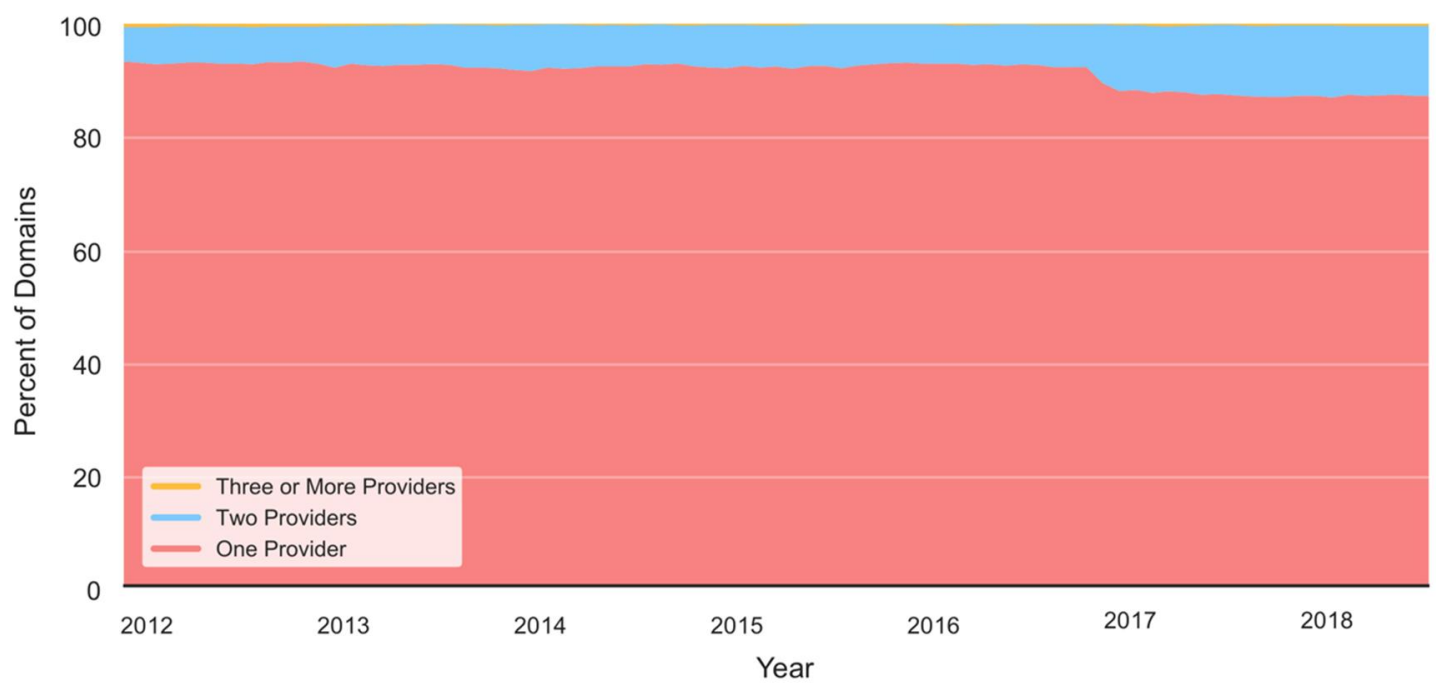

Figure 12. The percentage of domains that registered one, two, or three or more DNS providers.

Interestingly, diversification was more common amongst the top ranked 100 domains per month in the sample. The percentage of domains using a single provider decreased from $95 \%$ in November of 2011 to $87 \%$ in October 2016. After the Dyn attack, it dropped to a low of $79 \%$ in July $2018 .{ }^{17}$

opted to use multiple externally hosted DNS providers, while about $34.4 \%$ of diversified domains chose to employ a self-hosted domain nameserver and an externally hosted one.

${ }^{17}$ This tendency to stick with one DNS provider was even more pronounced amongst entrant domains. Entrant domains in the sample tended to diversify at considerably lower rates than original domains, with around 5\% diversifying for most of the timescale prior to the Dyn attack. They also reacted less intensely to the Dyn attacks than did the original domains: their diversification rate increased by about half as much. This may signal that entrants are more wedded to single-provider cloud hosting. In contrast to the original domains that were created before there were many cloud-based DNS providers and had to build their own DNS infrastructure, entrant domain owners have more external DNS providers to choose from. Moreover, external providers handle more than just DNS management, so choosing to host everything with one provider may be the easiest solution for entrants. 


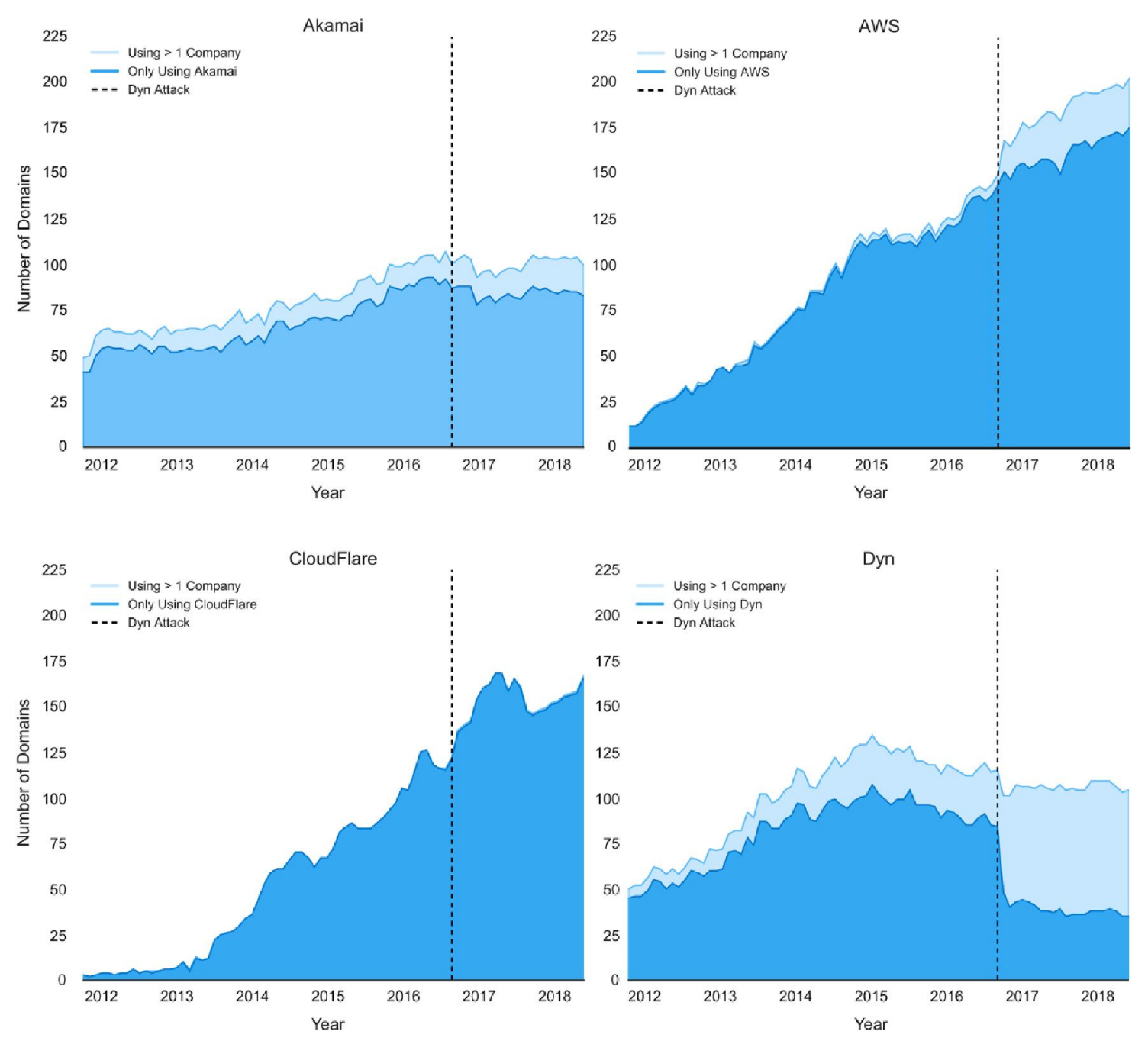

Figure 13. The number of undiversified and diversified domains using DNS services provided by one of the four largest external DNS providers: Akamai, AWS, Cloudflare, and Dyn.

There is a very significant degree of variation in patterns of diversification and growth among external DNS providers. As is evident from figure 13 showing the 4 largest externally hosted DNS providers in the sample as of July 2018, customers of some externally hosted DNS providers tended to diversify much more than those of others. A 
fluctuating percentage of $81 \%$ and $89 \%$ of Akamai users remained undiversified throughout the sampled timescale as Akamai's DNS service has roughly doubled in size. There was a steady increase in diversification rates among Dyn customers from November 2011 until the Dyn attack, at which point the percentage of undiversified domains using Dyn's services plummeted to $48 \%$.

Cloudflare maintained consistently near-zero diversification rates for the entire timescale, over the course of which it has exploded in size from having virtually no market share to becoming the second most dominant provider in the sample. This near-complete lack of diversification is a product of Cloudflare's security model, which requires that DNS traffic is routed through the Cloudflare network to protect against DDoS attacks and other network insecurities (Cloudflare, 2021). This approach may make it difficult for domains to register a secondary nameserver managed by a different DNS provider. Until the Dyn attack (after which many domains added AWS as a secondary DNS provider) AWS also registered an extremely low diversification rate - no more than $4 \%$ of domains using AWS diversified at any point before the Dyn attack. Between the attack and July 2018, the proportion of diversified domains using AWS increased to about $13 \%$.

\section{Why Not Diversify?}

Given the advantages of diversification, why would so many domain operators neglect to use multiple DNS providers? Configuring DNS across multiple DNS providers can be a non-trivial technical undertaking. When a DNS server responds to a request by a DNS resolver, it authenticates its response with a set of "NS records," which must accurately reflect all of a domain's nameservers (even across multiple providers) (Elz, R. et al., 1997). Using multiple DNS providers therefore requires that the domain's administrators be able to edit or otherwise synchronize NS records among DNS servers that are managed by different providers. 
Many large external DNS companies provide support and documentation for implementing DNS diversification, with some offering explicitly "secondary" DNS configurations as a service (Dyn, 2020). However, configuring multiple DNS providers can still require significant development efforts and the generation of (potentially insecure or buggy) bespoke code. For a company that does not have the same knowledge or technical resources, diversifying DNS providers may prove to be more trouble than it is worth (Hildrew \& Sivapalan, 2016).

The discrepancies in diversification indicated in the previous subsection likely point to differences in user experiences between external DNS providers which affect the ease with which domains can diversify among multiple DNS providers. It is worth noting that Dyn - which had the most diversified client domains of any large external DNS host following the October 2016 attack - provides specific guidelines for making its DNS services work smoothly with those of other providers (Dyn, 2020). Neustar (Neustar, 2017) and DNS Made Easy (DNS Made Easy, 2020), the runners up to Dyn in this regard, also provide such instructions. AWS, which falls behind every other provider except for CloudFlare in terms of the diversification of its clients, offers no such readily accessible support.

Moreover, it should be noted that large externally managed DNS providers including Dyn - generally have near-perfect uptime records, with most attaining uptime of $99.9 \%$ or higher ${ }^{18}$ on a monthly basis (Burt, 2015). The cost, difficulty, and potential technical issues associated with diversification among multiple providers may simply not be worthwhile for a majority of firms. As the saying goes, "Nobody ever got fired for buying IBM" (Maycotte, 2014).

However, as the number of insecure web-connected devices continues to grow at a breakneck pace, it is likely that the scale and frequency of botnet-driven DDoS attacks capable of compromising even the largest providers will continue to increase (Kapur,

$1899.9 \%$ uptime - "three nines" - is a common responsiveness goal for web domains. 
2017). If sophisticated externally managed DNS providers cannot scale their countermeasures accordingly, uptime figures for even the largest services may begin to decline.

\section{The Dyn Attack: A Wakeup Call (For Some)}

The Dyn attack highlighted the value of diversification among multiple DNS providers. At the time of the attack, 84 domains in our sample were using Dyn as their only DNS provider. Even more domains - including Netflix - were using Dyn as the sole DNS for alternate domains delivering content to their sites. The relationship between DNS vulnerability and CDNs should be explored further, but currently falls beyond the scope of this paper (Fastly, 2016).

Following the Dyn attack, a number of cybersecurity industry professionals and firms specializing in IT infrastructure offered vocal support for multi-provider DNS diversification (Kephart, 2016; Radware, 2016; York, 2016). Per the analysis above, a significant number of domains did diversify, bringing the percentage of domains in the sample using only one provider from 92.2\% in October 2016 to $87.1 \%$ in July 2018 . However, despite this coverage and evident shift in the DNS space, it seems that the lessons of the Dyn attack were learned primarily by those who suffered from them directly. Of the 55 domains that remained in the sample between October 2016 and July 2018 and diversified during that timeframe, the majority $(52.7 \%)$ were using Dyn alone prior to the attack. $^{19}$

It is unfortunate that the instructive value of the Dyn attacks seem to have been limited in large part to those who suffered from it. But even for those who aren't counted among the once bitten twice shy, the attack should serve as a reminder that DNS

\footnotetext{
${ }^{19}$ Of these Dyn users, more than two-thirds went to either AWS or NSOne, a relatively recent entrant into the DNS space which expanded its share somewhat in the wake of the Dyn attack.
} 
diversification can serve as a powerful corrective to the systemic cybersecurity risks imposed by DNS hosting market concentration.

\section{Conclusion}

A stable DNS infrastructure is critical to the operation of the Internet. Growing cybersecurity challenges from botnets, ransomware, and other service disruption systems will stress-test even the biggest providers, and future downtime from such attacks is all but inevitable. With downtime comes SEO penalties and the loss of revenue and user trust. In addition to this threat of bad actors, even the largest cloud services providers have shown themselves to be vulnerable to large service outages (Kapur, 2017).

The concentration of the DNS space makes confronting these challenges all the more urgent. As a larger and larger proportion of the Internet's biggest sites fall under the management of a small number of externally hosted DNS providers, single points of failure will continue to emerge and grow in magnitude. While every company with an Internet presence will ultimately have to weigh its costs and benefits, diversification promises a powerful means by which many of the dangers of this new DNS environment can be mitigated. All would be prudent to consider altering their DNS architecture accordingly.

Ultimately, the biggest determinant of domain owners' willingness to diversify may be the extent to which providers choose to support and encourage such diversification. If single service lock-in is the model of cloud DNS platforms, widespread diversification is unlikely. However, providers could encourage diversification of DNS management services by requiring domain owners to select a secondary DNS provider or even specify which secondary providers they should choose. If more domains adopt and support affordable and easily configurable "Secondary DNS" models such as that offered by Dyn, a diversified and resilient DNS space could easily come into reach. 
And yet, important questions remain. The data presented here suggest that administrators of prominent domains have adapted their DNS management strategies in a wide variety of ways. A growing majority have delegated DNS management to external providers, while a shrinking minority have elected to self-host. Some diversified their DNS infrastructure by way of secondary DNS providers in the wake of the Dyn attack, while others continued to work with a single provider -- even a number of those that had seen their sites go unreachable. These differences in decision making present an open question

for future research, one which goes beyond the scope of our dataset. Further analyses might investigate DNS management practices in terms of covariates not considered here, including industry affiliation, firms' technical sophistication, other characteristics of a domain's hosting configuration, and economic factors.

\section{Acknowledgements}

The authors would like to thank Hans Christian Gregersen and Matt Phillips for their work in preparing early versions of our dataset. The authors would also like to thank David Dinin and Andy Ellis for lending us their expertise in DNS and other technical matters.

\section{References}

Albert, R., Jeong, H., \& Barabási, A. (2000). Error and attack tolerance of complex networks. Nature, 406, 378-382. https://doi.org/10.1038/35019019

Alexa. Top Sites. http://www.alexa.com/topsites

Alexa. (2020). How are Alexa's traffic rankings determined? https://support.alexa.com/hc/en-us/articles/200449744-How-are-Alexa-s-trafficrankings-determined, archived at https://perma.cc/CF9U-Z9F8

[@awscloud]. (2017, Feb. 28). The dashboard not changing color is related to S3 issue. See the banner at the top of the dashboard for updates. [Tweet]. Twitter. https://twitter.com/awscloud/status/836656664635846656?lang=en Blitzer, D. (2013, July 9). Inside the S\&P 500: Selecting Stocks. Indexology Blog. 
http://www.indexologyblog.com/2013/07/09/inside-the-sp-500-selecting-stocks/, archived at https://perma.cc/HS8A-W273

Burt, C. (2015, January 8). Many DNS and CDN Services Attain Near Perfect Uptime: CloudHarmony Report. Whir Hosting | Cloud. https://perma.cc/8KMZ-JECH

DNS Made Easy. (n.d.). Secondary DNS Services. Retrieved October 10, 2020, from https://www.dnsmadeeasy.com/services/secondarydns/

Dyn. (2017). Explore DDoS. https://perma.cc/94ED-SZ9D

Dyn. (2020). Using External (Secondary) Nameservers. https://help.dyn.com/usingexternal-nameservers/, archived at https://perma.cc/M2LB-8E3V

Elz, R. et al. (1997). Selection and Operation of Secondary DNS Servers. RFC 2182. https://doi.org/10.17487/RFC2182

Fastly. (2016, October). Widespread Dyn DNS outage affecting Fastly customers. https://www.fastly.com/security-advisories/widespread-dyn-dns-outage-affectingfastly-customers, archived at https://perma.cc/P8B3-FD9N

Fishkin, R. (2015, June 2). The Traffic Prediction Accuracy of 12 Metrics from Complete, Alexa, SimilarWeb, \& More. Sparktoro. https://moz.com/rand/trafficprediction-accuracy-12-metrics-compete-alexa-similarweb/, archived at https://perma.cc/32SC-MBEU

Hildrew, S., \& Sivapalan, J. (2016, December 23). Multiple DNS: Synchronising Dyn to AWS Route 53 [The Guardian]. Developer Blog. https://www.theguardian.com/info/developer-blog/2016/dec/23/multiple-dnssynchronising-dyn-to-aws-route-53, archived at https://perma.cc/WDP4-MY93

How DDoS Attacks Can Impact Your SEO. (2013, December 24). DOSArrest. https://www.dosarrest.com/ddos-blog/how-ddos-attacks-can-impact-your-seo/, archived at https://perma.cc/E6G5-8KNQ

Kapur, N. (2017, January 13). The Rise of IoT Botnets. American Security Project. https://www.americansecurityproject.org/the-rise-of-iot-botnets/, archived at https://perma.cc/EMH7-CHL9

Kephart, N. (2016, October 25). The DDoS Attack on Dyn's DNS Infrastructure. Thousand Eyes. https://blog.thousandeyes.com/dyn-dns-ddos-attack/, archived at https://perma.cc/P3UL-KH8F

Le Pochat, V., Van Goethem, T., Tajalizadehkhoob, S., Korczynski, M., \& Joosen, W. (2019). Tranco: A Research-Oriented Top Sites Ranking Hardened Against 
Manipulation. Network and Distributed Systems Security (NDSS) Symposium 2019. https://www.ndss-symposium.org/wpcontent/uploads/2019/02/ndss2019_01B-3_LePochat_paper.pdf

Matsumoto, A., Merlone, U., \& Szidarovszky, F. (2011). Some notes on applying the Herfindahl-Hirschman Index. Applied Economics Letters, 19(2), 181-184. https://doi.org/10.1080/13504851.2011.570705

Matthews, T. (2014). What DDoS Attacks Really Cost Businesses. Incapsula.

Maycotte, H. O. (2014, December 9). Your Startup Dilemma: Nobody Ever Got Fired for Buying IBM. Forbes. https://www.forbes.com/sites/homaycotte/2014/12/09/yourstartup-delimma-nobody-ever-got-fired-for-buying-ibm/\#3bdcb7fa16b6, archived at https://perma.cc/PN7V-B5XF

New Report: The Case for a Secondary DNS Service. (2017, April 20). Neustar Blog. https://www.neustar.biz/blog/secondary-dns-service-paper, archived at https://perma.cc/Q7VN-ZY24

Newman, L. (2016, October 21). What We Know About Friday's Massive East Coast Internet Outage. Wired. https://perma.cc/3BU2-6F4K

Nozomi Networks. (2020). OT/IoT Security Report. https://info.nozominetworks.com/otiot-security-report-1h-lp-0

Perlroth, N. (2016, October 21). Hackers Used New Weapons to Disrupt Major Websites Across U.S.. New York Times. https://www.nytimes.com/2016/10/22/business/internet-problems-attack.html

Rhoades, S. (1993). The Herfindahl-Hirschman Index. Federal Reserve Bulletin, 79, $188-189$.

Tranco. (2020). Methodology. https://tranco-list.eu/methodology

York, D. (2016, October 25). How To Survive A DNS DDoS Attack-Consider using multiple DNS providers. Internet Society Blog. https://www.internetsociety.org/blog/2016/10/how-to-survive-a-dns-ddos-attackconsider-using-multiple-dns-providers/, archived at https://perma.cc/S3DMTYXK

Zittrain, J. (2013). Will the Web Break? Philosophical Transactions of the Royal Society A, 371, 386-394. http://dx.doi.org/10.1098/rsta.2012.0386 